\title{
Tescalcin is an essential factor in megakaryocytic differentiation associated with Ets family gene expression
}

\author{
Konstantin Levay ${ }^{1}$ and Vladlen Z. Slepak ${ }^{1,2}$
}

1Department of Molecular and Cellular Pharmacology and 2Neuroscience Program, University of Miami Miller School of Medicine, Miami, Florida, USA.

\begin{abstract}
We show here that the process of megakaryocytic differentiation requires the presence of the recently discovered protein tescalcin. Tescalcin is dramatically upregulated during the differentiation and maturation of primary megakaryocytes or upon PMA-induced differentiation of $\mathrm{K} 562$ cells. This upregulation requires sustained signaling through the ERK pathway. Overexpression of tescalcin in K562 cells initiates events of spontaneous megakaryocytic differentiation, such as expression of specific cell surface antigens, inhibition of cell proliferation, and polyploidization. Conversely, knockdown of this protein in primary $\mathrm{CD}^{3} 4^{+}$hematopoietic progenitors and cell lines by RNA interference suppresses megakaryocytic differentiation. In cells lacking tescalcin, the expression of Fli-1, Ets-1, and Ets-2 transcription factors, but not GATA-1 or MafB, is blocked. Thus, tescalcin is essential for the coupling of ERK cascade activation with the expression of Ets family genes in megakaryocytic differentiation.
\end{abstract}

\section{Introduction}

Hematopoiesis is a multistage developmental process maintained by a limited number of hematopoietic stem cells that proliferate, self renew, and differentiate into mature blood cells of all lineages. In the past, studies of purified primary cells, as well as animal and cell line models that have preserved part of the differentiation control mechanism, helped to define the major stages of this fundamental process. While all the intermediate steps have yet to be uncovered, it is now clear that differentiation requires kinasemediated signaling from various extracellular stimuli to transcription factors in the nucleus. Previous studies demonstrated that activation of ERK signaling pathway promotes hematopoietic differentiation and commitment. For example, ERK regulates T cell development by favoring commitment to the CD4 lineage $(1,2)$. In some myeloid cell lines, activation of ERK induces differentiation into monocytes (3). In K562, HEL, CMK, and UT7 cell lines the ERK pathway was shown to regulate commitment between megakaryocytic and erythroid cell fates. Activation of ERK in these cell lines leads to the suppression of erythroid markers and induces many features of megakaryocytic differentiation, such as growth arrest, expression of specific markers, increased cell adhesion and spreading, cellular enlargement, and polyploidization (4-8).

Activated ERK translocates to the nucleus and phosphorylates several transcription factors, leading to both temporal and spatial changes in gene expression. One group of such nuclear effectors of ERK signaling is the Ets family of transcription factors, which is highly conserved from metazoans to humans $(9,10)$. Ets proteins are required for the expression of growth factors and their cognate receptors as well as other components of the signaling pathways responsible for the induction of subsequent stages of

Nonstandard abbreviations used: BFU-MK, burst-forming unit-MK; GPIIb, glycoprotein IIb; MK, megakaryocyte; RQ-PCR, real-time quantitative PCR analysis; shRNA, short hairpin RNA; TPO, thrombopoietin.

Conflict of interest: The authors have declared that no conflict of interest exists. Citation for this article: J. Clin. Invest. 117:2672-2683 (2007). doi:10.1172/JCI27465. differentiation, including members of their own family $(11,12)$. The exact mechanisms regulating the expression of Ets genes are not yet fully understood.

Among the members of Ets family, Fli-1 and Ets-1 are particularly important in megakaryocytopoiesis. They transactivate expression of many lineage-specific genes, including thrombopoietin (TPO) receptor c-MPL and glycoprotein Ib $\alpha$ (GPIb $\alpha$ ), GPIIb, GPV, GPVI, GPIX, von Willebrand factor, and platelet factor 4 (13-25). Ets-1 regulates both the tissue-specific expression and DNA-binding activity of AML-1/RUNX1 transcription factor, which is required for megakaryocytic maturation (26-28). Targeted disruption of Fli-1 in transgenic mice results in megakaryocytopoietic deficiencies and profound cerebrospinal hemorrhage, followed by death at E11.5 (29, $30)$. In humans, the hemizygous loss of FLI1 gene underlies ParisTrousseau/Jacobsen syndrome, an acute bleeding disorder (31).

Tescalcin was discovered as an autosomal gene expressed during the early stages of mouse testis development (32). It encodes a 24-kDa EF-hand protein with a single functional $\mathrm{Ca}^{2+}$-binding site that can bind $\mathrm{Ca}^{2+}$ with micromolar affinity (33). In vitro studies implicate tescalcin in the inhibition of the phosphatase activity of calcineurin $\mathrm{A}$ as well as in the regulation of $\mathrm{Na}^{+} / \mathrm{H}^{+}$exchanger via a direct protein-protein interaction (33-35). Tescalcin has a restricted tissue distribution pattern with the highest protein expression level in the adult mouse heart, brain, and stomach as well as in primary human blood cells and hematopoietic cell lines. Its mRNA is also detected in mouse bone marrow and fetal liver. The function of tescalcin in these tissues is not known, but its expression in developing tissues suggests a role in differentiation.

Here we present evidence that tescalcin is indispensable for the expression of Ets family transcription factors, which regulate differentiation of progenitor cells along the megakaryocytic lineage.

\section{Results}

Upregulation of tescalcin correlates with megakaryocytic differentiation. We previously showed that tescalcin is expressed in some hematopoietic cell lines, including chronic myelogenous leukemia K562 cells (33). 


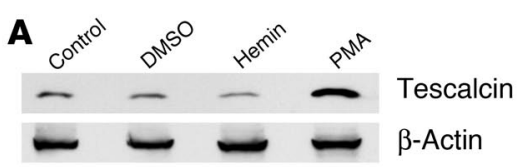

D
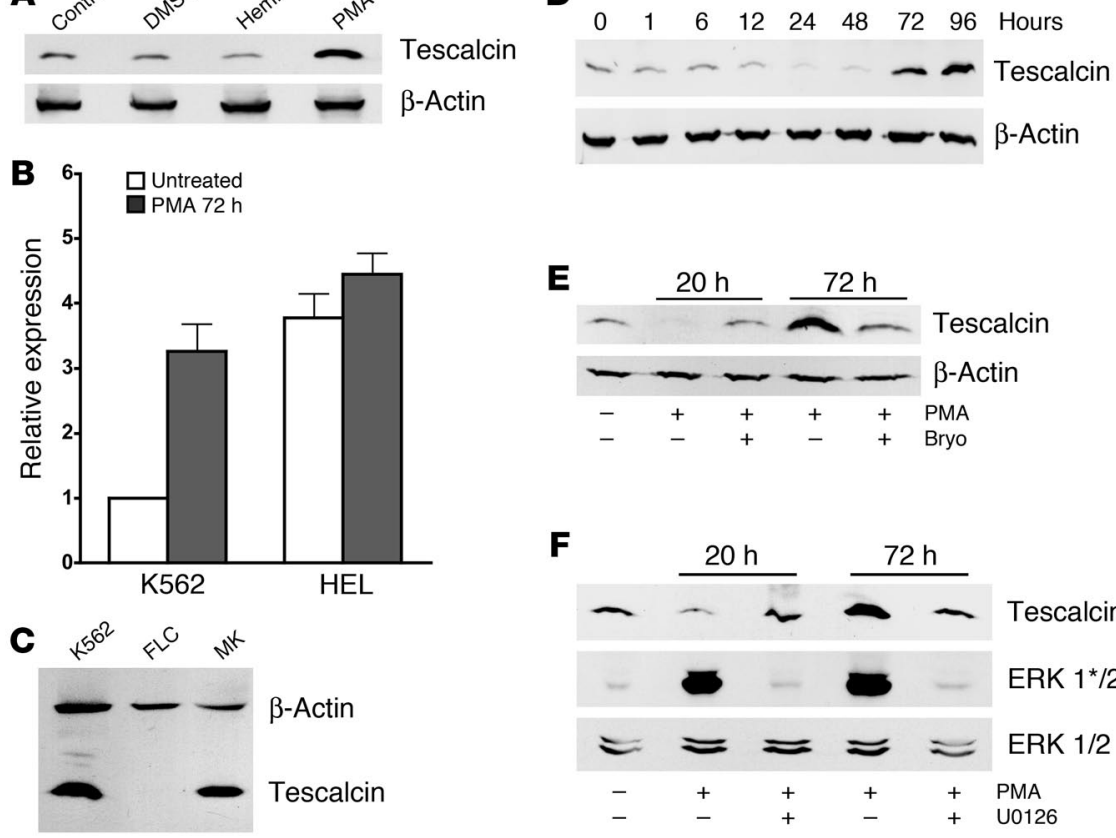

Figure 1

The expression of tescalcin is increased during megakaryocytic differentiation. (A) K562 cells were cultured in the presence of DMSO, hemin, or PMA for 72 hours, as described in Methods. Cells lysates were subjected to Western blot analysis with antibody against tescalcin. (B) Stimulation with PMA upregulates tescalcin at the mRNA level. K562 and HEL cells were cultured in the absence or presence of PMA for 72 hours. Total RNA subjected to quantitative RQ-PCR with tescalcin-specific primers (TaqMan). Obtained values $(n=3$; mean $\pm \mathrm{SD})$ were normalized to $18 \mathrm{~S}$ ribosomal RNA, and expressed relative to unstimulated K562. (C) Tescalcin expression in primary MKs. Mature mouse MKs were obtained from fetal livers as described in Methods. Lysates of fetal liver cells (FLC) and MKs were analyzed for tescalcin expression by Western blot. K562 lysate was used as positive control. (D) K562 cells were cultured in the presence of PMA (10 nM) for the indicated times, and the accumulation of tescalcin was determined by Western blot. (E) Bryostatin blocks PMA-induced upregulation of tescalcin. K562 cells were stimulated by $10 \mathrm{nM}$ PMA in the absence or presence of $100 \mathrm{nM}$ bryostatin (Bryo). The expression of tescalcin was determined by Western blot. (F) Sustained ERK activity is required for tescalcin upregulation. K562 cells were stimulated by PMA $(10 \mathrm{nM})$ in the absence or presence of MEK1/2 inhibitor ( $20 \mu \mathrm{M}$; U0126). Cell lysates were probed with antibodies to tescalcin, total p44/42 MAPKs (ERK1/2), and phospho-p44/42 MAP kinases (ERK1*/2*).

K562 cells are regarded as pluripotent hematopoietic progenitors that express specific markers of granulocytic, monocytic, erythroid, and megakaryocytic lineages. In addition, K562 cells can be induced to differentiate in vitro following stimulation by a variety of specific agents (36). To determine whether tescalcin is regulated in hematopoietic differentiation, K562 cells were stimulated with 1.5\% DMSO, $30 \mu \mathrm{M}$ hemin, or $10 \mathrm{nM}$ PMA to promote granulocytic, erythroid, or megakaryocytic differentiation, respectively. Treatment with PMA, but not with DMSO or hemin, led to a dramatic increase in the level of tescalcin protein (Figure 1A). A slight increase in tescalcin expression was also observed in PMA-treated HEL cells (data not shown), another pluripotent hematopoietic cell line expressing markers of megakaryocytic differentiation $(37,38)$. Real-time quantitative PCR analysis (RQ-PCR) on total RNAs isolated from K562 and HEL cells demonstrated that PMA-induced upregulation of tescalcin was at least partially due to an increase in the accumulation of its mRNA (Figure 1B). We also noticed that the level of tescalcin mRNA in unstimulated HEL cells was at least 4-fold higher than in K562 (Fig- ure 1B). Accordingly, HEL cells expressed higher levels of megakaryocytic markers (37). To find out whether tescalcin is expressed in primary cells, we used an established method to obtain mature megakaryocytes (MKs) from mouse fetal liver cells stimulated by TPO (39). Western blot analysis showed that while tescalcin was not detected in fetal liver cells, it was highly expressed in terminally differentiated MKs (Figure 1C).

Upregulation of tescalcin requires sustained ERK activity. Stimulation with PMA causes the activation of PKC, which in K562 cells can lead to a number of differentiation events including the acquisition of megakaryocytic markers, growth arrest, and development of polyploid nuclei $(4,40)$. It has been previously shown that irreversible commitment of K562 cells to the megakaryocytic lineage depends on sustained (>24 hours) ERK activity and can be reached by treatment with PMA (6). We found that there was a delay of at least 48 hours between initiation of stimulus and the rise in tescalcin accumulation (Figure 1D). This expression pattern parallels the PMAinduced expression of other factors involved in megakaryocytopoiesis (41) and indicates that tescalcin may be a part of the differentiation program. To test this idea, we treated cells with bryostatin, a structurally distinct PKC activator. Unlike PMA, bryostatin is only capable of transient activation of ERK, which is insufficient to induce differentiation. Moreover, bryostatin effectively blocks PMA-induced differentiation in K562 cells (40). In our experiments, bryostatin inhibited upregulation of tescalcin induced by PMA (Figure 1E). To determine whether ERK activity is necessary for PMA-induced upregulation of tescalcin, we used U0126, a highly selective inhibitor of both MEK1 and-2, known direct upstream regulators of ERK. Pretreatment of cells with $20 \mu \mathrm{M}$ U0126 effectively blocked both the activation of ERK and upregulation of tescalcin in response to PMA (Figure 1F).

Altogether, our results suggest that upregulation of tescalcin is primarily dependent on sustained ERK activity and correlates with the induction of megakaryocytic differentiation.

K562 cells overexpressing tescalcin recapitulate the events of early megakaryocytic differentiation. To establish the cause and effect relationship between upregulation of tescalcin and megakaryocytic differentiation, we generated several stable clones of K562 cells with constitutive overexpression of tescalcin. Cells transfected with empty pcDNA3 vector were used as negative controls. For the biochemical and functional studies and to avoid clonal variability, we pooled 3 independent clones of tescalcin overexpressors and corresponding controls. These pools of transfectants were designated $\mathrm{K} 562-\mathrm{Tsc}(+)$ and $\mathrm{K} 562-\mathrm{Ctrl}(+)$, respectively.

We noticed that upon overexpression of tescalcin the fraction of enlarged K562 cells had considerably increased. This observation 

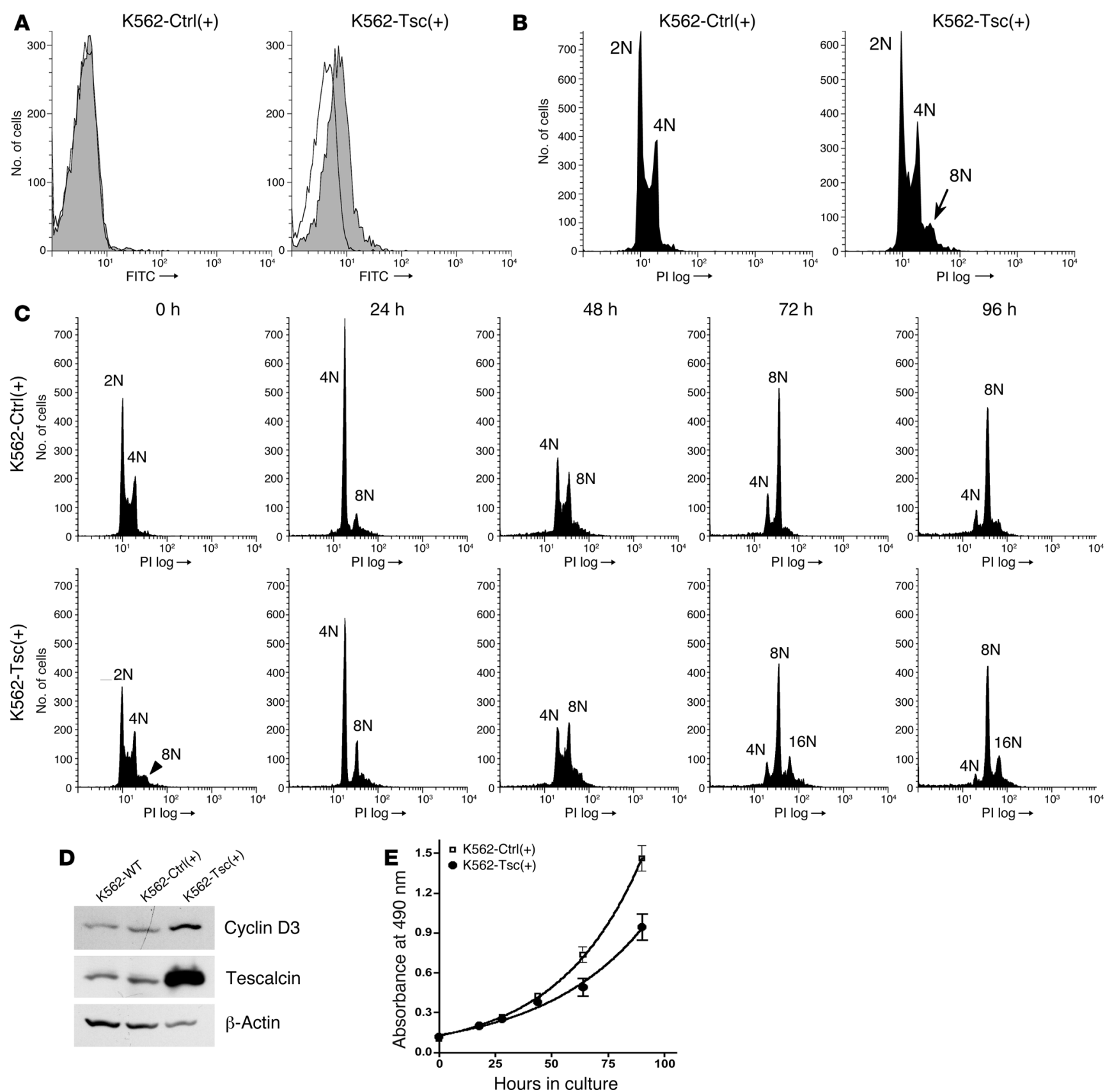

Figure 2

Overexpression of tescalcin in K562 cells induces events of early megakaryocytic differentiation. (A) Overexpression of tescalcin leads to an increased level of surface GPIlb. Control [K562-Ctrl(+)] and tescalcin overexpressing [K562-Tsc(+)] cells were stained with FITC-conjugated CD41-specific antibody. Samples were analyzed using BD FACScan, with a minimum of 10,000 events acquired per sample. Expression profile of surface CD41 in $\mathrm{K} 562-\mathrm{Ctrl}(+)$ and $\mathrm{K} 562-\mathrm{Tsc}(+)$ cells is shown as filled histogram. FITC-conjugated isotype mouse IgG was used as negative control (open histogram). (B) Overexpression of tescalcin promotes polyploidy in K562 cells. K562-Ctrl(+) and K562-Tsc(+) cells were fixed and stained with propidium iodide and their DNA content was analyzed by FACS. PI log, propidium iodide, logarithmic scale. (C) The onset of PMAinduced polyploidy occurs faster in tescalcin-overexpressing cells. K562-Ctrl(+) and K562-Tsc(+) cells were cultured in the presence of PMA for indicated times and analyzed as described in B. (D) Cyclin D3 accumulation in tescalcin-overexpressing cells is increased. Wild-type [K562-WT], $\mathrm{K} 562-\mathrm{Ctrl}(+)$, and $\mathrm{K} 562-\mathrm{Tsc}(+)$ cell lysates were probed with cyclin D3- and tescalcin-specific antibodies. $\beta$-Actin was used as a loading control. (E) Reduction of proliferation rate in tescalcin-overexpressing cells. Absorbance at $490 \mathrm{~nm}$ of K562-Ctrl(+) and K562-Tsc(+) cell lines were compared in the MTS-based cell proliferation assay, as described in Methods. Data represent 3 independent experiments (mean \pm SD).

led us to the idea that high levels of tescalcin in K562 may promote spontaneous cell differentiation along megakaryocytic lineage. To test this hypothesis, we examined typical characteristics of mega- karyocytic differentiation, such as the expression of GPIIb, polyploidy, and the rate of cell proliferation (Figure 2, A-E). The expression of GPIIb, which is a part of GPIIb/IIIa (also known as $\alpha I I b \beta I I I$, 
A

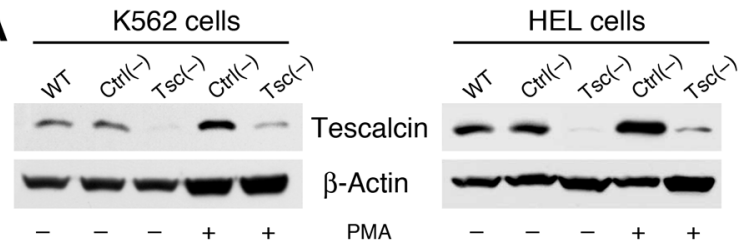

B
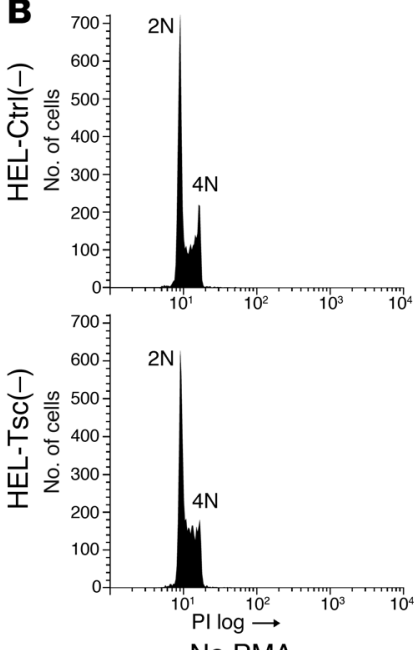

No PMA

C

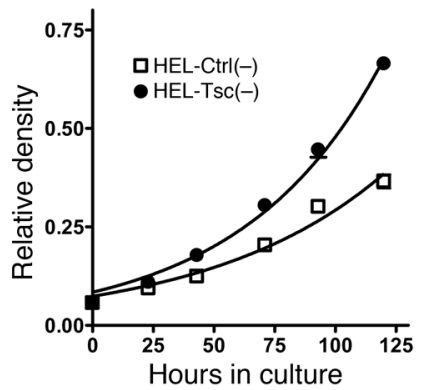

CD41/CD61) integrin complex, on the cell surface is one of the key events of early megakaryocytopoiesis. Flow cytometry analysis confirmed that K562-Tsc(+) cells show increased level of GPIIb and that such an increase is mostly found on a subpopulation of larger cells (Figure 2A and Supplemental Figure 1; supplemental material available online with this article; doi:10.1172/JCI27465DS1).

Another indicator of megakaryocytic differentiation is polyploidization, which is essential for the efficient production and release of platelets. It is known that in response to PMA stimulation, K562 nuclear DNA ploidy rises to $4 \mathrm{~N}-16 \mathrm{~N}$ concurrently with an increase in cell volume (36). Using FACS analysis of propidium iodide-stained cells, we compared the DNA content in $\mathrm{K} 562-\mathrm{Ctrl}(+)$ and $\mathrm{K} 562-\mathrm{Tsc}(+)$ cells. In unstimulated K562-Tsc(+) cells the number of cells with DNA content of $8 \mathrm{~N}$ and more was increased to $8 \%-10 \%$ compared with less than $1 \%$ in controls (Figure $2 \mathrm{~B}$ ). Further, we found that high levels of tescalcin caused accelerated onset of polyploidy in response to PMA. More than $20 \%$ of $2 \mathrm{~N}$ and $4 \mathrm{~N}$ cells overexpressing tescalcin shifted to $8 \mathrm{~N}$ and higher ploidy after only 24 hours of stimulation. After 72 hours of PMA stimulation, a significant number of $\mathrm{K} 562-\mathrm{Tsc}(+)$ cells were already shifted to $16 \mathrm{~N}$ and higher ploidy, while the majority of control K562-Ctrl(+) cells remained at $4 \mathrm{~N}$ and $8 \mathrm{~N}$ (Figure 2C). It was reported earlier that the G1-phase cyclin, cyclin D3,

\section{Figure 3}

Tescalcin knockdown inhibits PMA-induced polyploidy and increases cell proliferation. (A) Western blot analysis of wild-type cells and cells expressing scrambled Ctrl(-) and tescalcin-specific Tsc(-) target sequence shRNAs. Cells were cultured in the absence and presence of PMA and then analyzed by Western blot with antibody to tescalcin. $\beta$-Actin was used as a loading control. (B) Comparison of the DNA content in control HEL-Ctrl(-) and tescalcin knockdown HELTsc(-) cells prior to or after stimulation by PMA for 72 hours. Cells were fixed, stained with propidium iodide, and analyzed by FACS. (C) Increase in cell proliferation after tescalcin knockdown. HEL-Ctrl(-) and HEL-Tsc(-) cells were compared in the MTS-based cell proliferation assay, as described in Methods. Data represent 3 independent experiments (mean $\pm S D$ ).

is upregulated in polyploidizing megakaryocytic cells and that overexpression of cyclin D3 in transgenic mice leads to increased MK number and ploidy $(42,43)$. In agreement with these earlier observations, we found that K562-Tsc(+) cells had an increased accumulation of cyclin D3 (Figure 2D). Next we determined the effect of tescalcin overexpression on the growth rate of K562 cells. For this we cultured cells in low-serum growth medium (1\% FBS) in order to decrease the proliferative drive while preserving viability. As expected of differentiating cells undergoing polyploidy and growth inhibition, proliferation of K562-Tsc(+) cells was significantly reduced (Figure 2E). Consistent with this observation, our attempt to generate stable constitutive overexpression of tescalcin in HEL cells resulted in a rapid cell enlargement and death within several days (Supplemental Figure 2).

Thus our results strongly indicate that overexpression of tescalcin is sufficient to induce the early events of megakaryocytic differentiation in K562 cells, including the expression of the MKspecific marker GPIIb, increased cell size, polyploidization, and inhibition of growth rate.

Effect of tescalcin knockdown on cell proliferation and PMA-induced polyploidy. To determine whether tescalcin is necessary for megakaryocytic differentiation, we inhibited its expression in K562 and HEL cells by vector-based short hairpin RNA (shRNA). In a number of generated stable clones the expression of tescalcin was dramatically reduced. Pools of 3 independent clones of each type, termed "K562-Tsc(-)" and "HEL-Tsc(-)," were used in all further experiments. PMA stimulation could cause only a minor increase of tescalcin expression in these cells (Figure 3A). For a negative control, we established several cell lines stably transfected with the shRNA construct encoding a scrambled target sequence and termed them "K562-Ctrl(-)" and "HEL-Ctrl(-)." The level of tescalcin expression in control cells was similar to wild-type K562 and HEL.

The phenotype caused by tescalcin knockdown was exactly opposite that observed with tescalcin overexpression. As expected, control HEL-Ctrl(-) cells responded to PMA treatment, with the DNA content rising up to $16 \mathrm{~N}$ ploidy level within 72 hours, whereas the DNA content in tescalcin knockdown HEL-Tsc(-) cells did not increase beyond 4N (Figure 3B). The DNA histogram for the uninduced HEL-Tsc(-) shows the accumulation of cells in $S$ phase of cell cycle, indicative of their higher proliferative activity. Indeed, the proliferation rate of HEL-Tsc(-) was significantly higher than that of HEL-Ctrl(-) cells (Figure 3C). Thus knockdown of tescalcin prevents HEL cells from undergoing PMA-induced polyploidy and increases the rate of cell proliferation.

Effects of tescalcin knockdown on adhesion of HEL cells. Adhesion and spreading on extracellular matrix is an important step in 

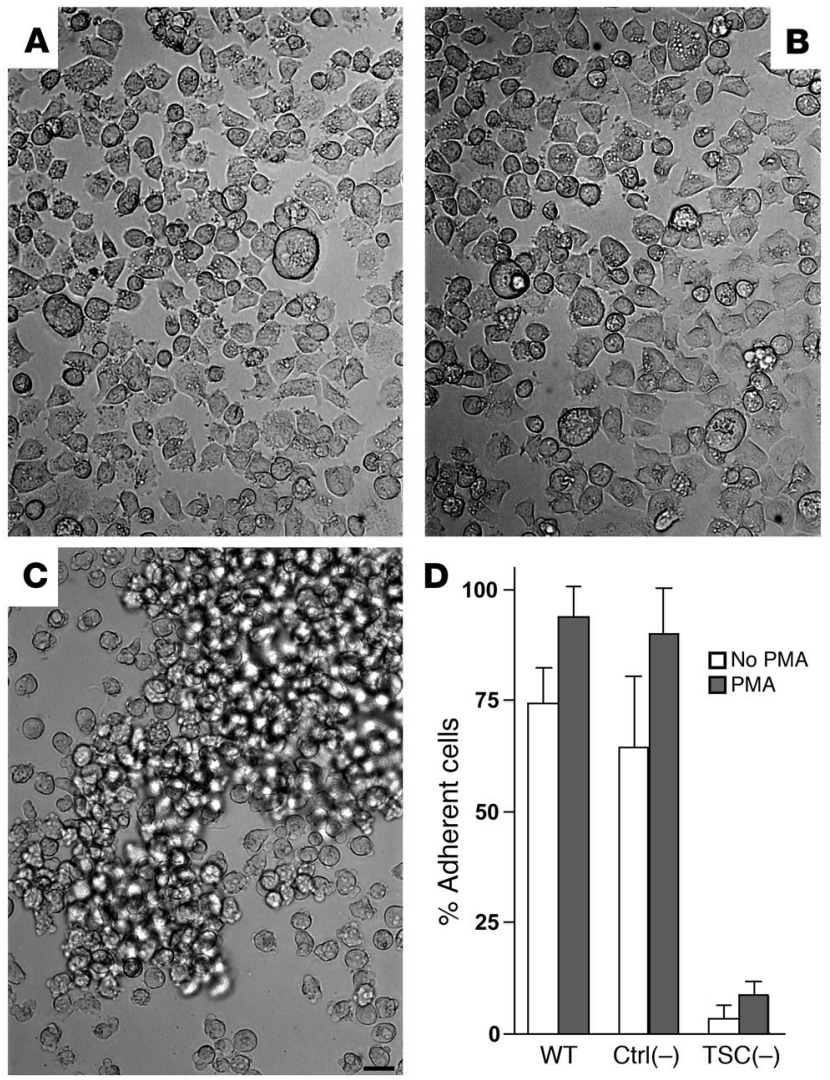

megakaryocytic differentiation and maturation in vivo (44). We examined the effect of tescalcin knockdown on fibronectin-specific adhesion. While HEL cells normally grow in suspension, they also strongly adhere to a plastic surface after treatment with PMA (Figure 4, A and B). Knockdown of tescalcin significantly reduced this ability. Instead we observed a homotypic aggregation of HELTsc(-) cells, which started approximately 36 hours after induction with PMA (Figure 4C). Moreover, fibronectin-specific adhesion was strongly inhibited as well. Under normal growing conditions $75 \%-80 \%$ of either HEL-WT or HEL-Ctrl(-) cells attached to fibronectin-coated plates within 30 minutes. The adherence increased to almost $100 \%$ when cells were stimulated with 10 nM PMA. In contrast, only about $3 \%$ of unstimulated HEL-Tsc(-) cells adhered to fibronectin, and the number of adhering cells did not increase above $12 \%$ even in the presence of PMA (Figure 4D).

Tescalcin is necessary for the expression of integrins. The major cell surface receptors for extracellular matrix molecules are integrins, a family of $\alpha \beta$ heterodimeric transmembrane glycoproteins. Integrins of $\beta_{1}$ and $\beta_{3}$ subfamilies, primarily $\alpha 4 \beta 1, \alpha 5 \beta 1$, and $\alpha \operatorname{IIb} \beta 3$, have been identified as being responsible for the binding of hematopoietic progenitor cells to fibronectin $(45,46)$. We used FACS analysis to assess the surface expression of GPIIb, $\alpha 4$, and $\alpha 5$ integrins which are known to be present in HEL cells. Consistent with previous reports, PMA treatment of HEL-Ctrl(-) resulted in a dramatic increase of GPIIb surface expression, whereas the expression of $\alpha 4$ and $\alpha 5$ integrins did not change significantly (Figure 5A). In tescalcin knockdown HEL-Tsc(-) cells, the surface expression of GPIIb and $\alpha 4$ molecules was below detectable levels either before or after PMA stimulation. This finding evidently explains the low

\section{Figure 4}

Tescalcin knockdown inhibits cell adherence to plastic and extracellular matrix proteins. Plated in plastic culture dishes, HEL-WT (A), HELCtrl(-) (B), and HEL-Tsc(-) (C) cells were stimulated by $10 \mathrm{nM}$ PMA. Cells were photographed 36 hours later at $\times 200$ magnification. Scale bar: $25 \mu \mathrm{m}$. (D) HEL-WT, HEL-Ctrl(-), HEL-Tsc(-) cells were allowed to adhere for 30 minutes in a 96-well culture plate precoated with fibronectin in the absence or presence of $10 \mathrm{nM}$ PMA. Adhesion was measured as described in Methods. Bar graphs represent results of 3 independent experiments performed in triplicate (mean $\pm S D$ ).

adhesion phenotype displayed by HEL-Tsc(-) cells (Figure 4). In contrast, the expression level of $\alpha 5$ integrin did not differ from that of control cells, indicating that the effect of tescalcin knockdown in HEL cells is specific to GPIIb and $\alpha 4$ integrin subunits. A similar effect of tescalcin knockdown on GPIIb expression was observed in K562-Tsc(-) cells (Figure 5B). Western blot analysis of total cell lysates demonstrated that not only surface expression but also the total level of GPIIb before and after PMA stimulation were drastically affected by the knockdown of tescalcin in both HEL and K562 cells (Figure 5, C and D).

Downregulation of tescalcin in human bone marrow $\mathrm{CD}_{3} 4^{+}$cells inhibits megakaryocytic differentiation. To examine the role of tescalcin in primary cells, we knocked down tescalcin in human bone marrow $\mathrm{CD}_{4}{ }^{+}$progenitors (Stem Cell Technologies Inc.) and tested their ability to differentiate using a CFU assay. Cells were transduced with lentiviral vectors encoding scrambled and tescalcin-specific shRNA. According to GFP coexpression, approximately $50 \%$ of cells were infected at MOI $=5$. The increase in MOI resulted in adverse effects on cell viability compared with noninfected CD $34^{+}$ cells. To assess the extent of tescalcin knockdown, infected cells were cultured in liquid MK differentiation medium for 7 days and analyzed by Western blot. Tescalcin level was reduced approximately 2 -fold in the entire pool of cells, indicating that knockdown was quite efficient in infected cell population (Figure 6A). The collagen-based CFU-MK analysis of the transduced CD34 ${ }^{+}$ progenitors revealed the following effects: the strongest effect of tescalcin knockdown was the 5-fold increase in frequency of small (3-20 cells/colony) CFU-MK-derived colonies (Figure 6, B and C). Importantly, the majority of CFU-MK colonies in the control consisted of 7-20 GPIIb-positive cells, whereas CFU-MK colonies after tescalcin knockdown contained only 3-5 cells (Figure 6, D and $E$ ). Another distinct effect of tescalcin knockdown was a 2-fold $(P=0.044)$ decrease in a number of large colonies resembling the primitive burst-forming unit-MK (BFU-MK). The number of well defined colonies of other hematopoietic cells (negative for GPIIb) increased more than 3-fold $(P=0.017)$ upon tescalcin knockdown (Figure 6, B and C). These results show that tescalcin plays an important role in megakaryocytic differentiation of primary bone marrow $\mathrm{CD}_{34}{ }^{+}$progenitors.

Downregulation of tescalcin inbibits GPIIb gene transcription. To determine whether tescalcin inhibits the expression of GPIIb at the mRNA or protein level, we analyzed the relative expression of GPIIb mRNA in HEL and K562 cells. RQ-PCR with TaqMan set of probe and primers (Applied Biosystems) showed that tescalcin knockdown in HEL-Tsc(-) results in almost complete inhibition of GPIIb expression (Figure 7A). Such a reduction of GPIIb mRNA level in cells lacking tescalcin can be explained by either lower transcriptional activity of GPIIb promoter or by mRNA instability. To assess the transcriptional activity of the full-length human GPIIb 

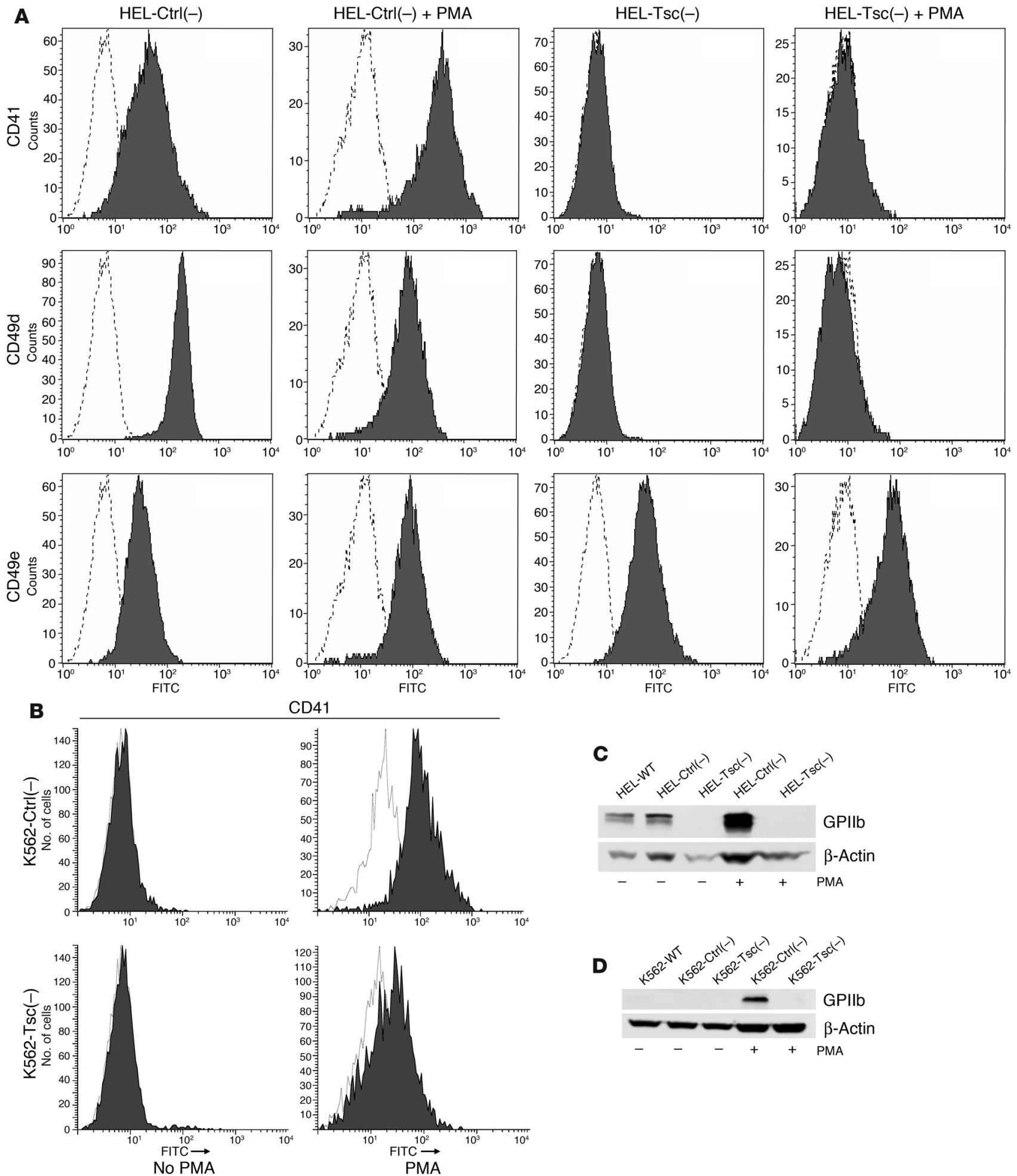

\section{Figure 5}

Tescalcin knockdown inhibits the expression of MK-specific marker GPIlb. (A) HEL-Ctrl(-) and HEL-Tsc(-) cells were cultured in the absence or presence of $10 \mathrm{nM}$ PMA for 72 hours and then analyzed for the cell surface expression of GPIIb (CD41), $\alpha 4$ (CD49d), and $\alpha 5$ (CD49e) integrins by FACS. (B) K562-Ctrl(-) and K562-Tsc(-) cells were cultured in the absence or presence of PMA for 72 hours and analyzed for the cell surface expression of GPIlb by FACS. (C) HEL-Ctrl(-) and HEL-Tsc(-) cells were cultured in the absence or presence of PMA for 72 hours and then subjected to Western blot analysis of the total expression of GPIlb. $\beta$-Actin was used as a loading control. (D) K562 cells were analyzed as described in $\mathbf{C}$. 

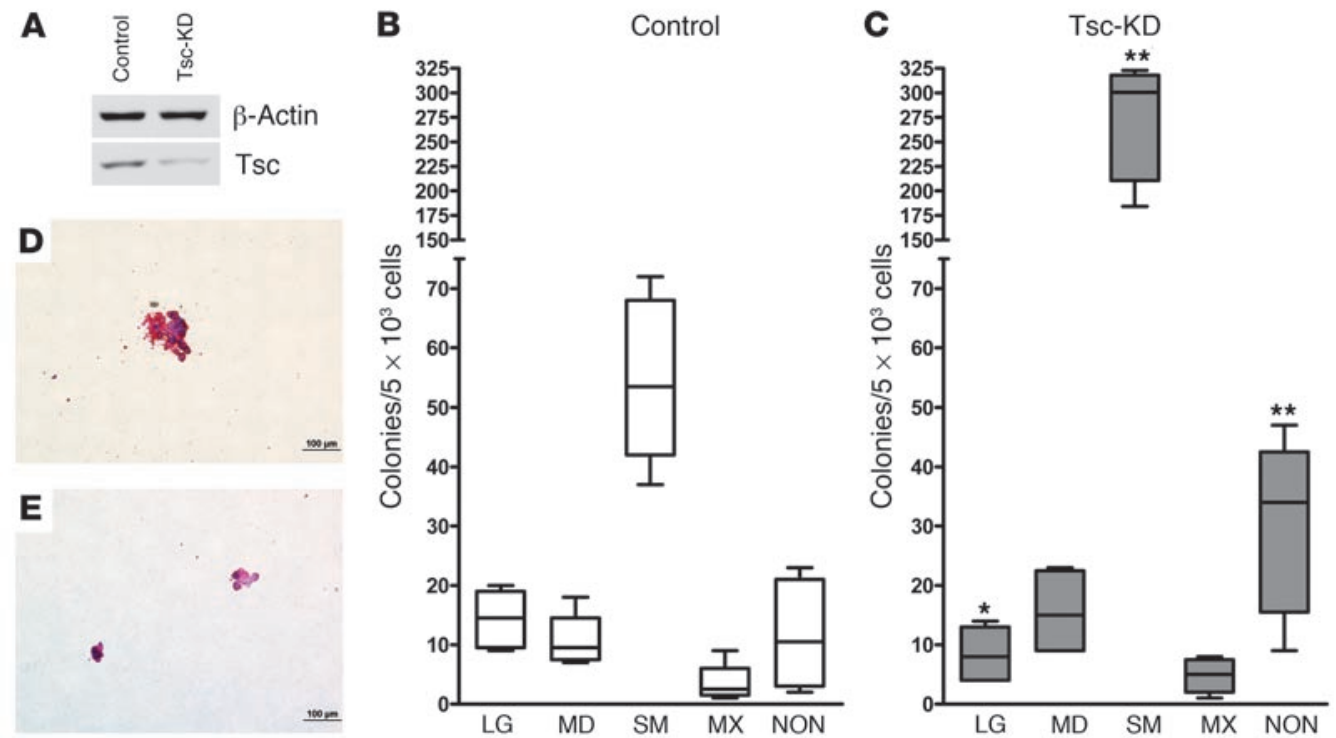

\section{Figure 6}

Silencing of tescalcin in CD34+ progenitors inhibits megakaryopoiesis. (A) $1 \times 10^{4}$ human bone marrow CD34+ cells were transduced with control or tescalcin-specific shRNA lentiviral vectors at $\mathrm{MOI}=5$, cultured in liquid differentiation media (MegaCult; StemCell Technologies Inc.) with cytokines ( $50 \mathrm{ng} / \mathrm{ml} \mathrm{TPO}, 10 \mathrm{ng} / \mathrm{ml} \mathrm{IL-6,} 10 \mathrm{ng} / \mathrm{ml} \mathrm{IL-3)} \mathrm{for} 7$ days, and analyzed for tescalcin expression by Western blot. (B and C) CD34+ cells were transduced by spinoculation with scrambled control (B) or tescalcin-specific shRNA (C) lentiviral vectors at MOI $=5$ in serum-free medium, as described in Methods. After recovering for 16 hours, cells were suspended in collagen-based MK differentiation media and plated in chamber slides. Twelve days later, cells were fixed, stained with anti-GPIlb antibody, and scored for 5 types of colonies. Large BFU-MK colony (LG) was defined as a cluster of 50-200 and more MKs with 2 or more different foci of development. Medium BFU-MK colony (MD) was defined as a cluster of 21-50 MKs. Small CFU-MK colony (SM) contained 3-20 MKs. Mixed (MX) colony was defined as a cluster of 2 or more MKs mixed with other hematopoietic cells. Non-MK (NON) colony was defined as cell cluster negative for GPIlb staining. Results of 6 experiments are presented as box-and-whisker plots, with the line within the box indicating the median, the box representing $25 \%$ and $75 \%$ percentiles, and whiskers indicating maximum and minimum values ( ${ }^{\star} P<0.05 ;{ }^{* *} P<0.005$, compared with scrambled control). (D and E) Typical small CFU-MK colonies formed after transduction with control (D) and tescalcin-specific (E) shRNA lentiviral vectors.

promoter we used a luciferase reporter assay. The GPIIb promoter was highly active in the untreated HEL-Ctrl(-) and less so in K562$\mathrm{Ctrl}(-)$ cells, consistent with the higher basal expression of tescalcin in HEL cells. However, in both HEL-Tsc(-) and K562-Tsc(-) cells, the activity of the promoter was blocked (Supplemental Figure 3). Therefore, our results show that the downregulation of tescalcin strongly inhibits GPIIb gene transcription.

Tescalcin is necessary and sufficient for the expression of Ets family genes. The expression of GPIIb mRNA in megakaryocytopoiesis is known to be controlled by a number of transcriptional factors, such as GATA-1, Ets-1, Ets-2, Fli-1, PU.1, MafB, and others $(41,47-51)$. We used RQ-PCR to quantitatively examine the expression of these factors in cells with both knocked down and overexpressed tescalcin.

Our results demonstrate that the levels of Ets- 1 and Fli- 1 mRNA in HEL-Tsc $(-)$ cells were almost undetectable. Ets-2 and PU.1 mRNAs were downregulated 10- and 4-fold, respectively, by tescalcin knockdown. The levels of GATA-1 and MafB mRNAs were not affected (Figure 7A), indicating the specificity of the tescalcin knockdown effect. Knockdown of tescalcin in K562 had a similar effect on expression of Ets family genes (data not shown). Consistent with the RQ-PCR data, Western blot analysis confirmed the decrease of Ets-1 and Fli-1 protein levels in cells lacking tescalcin. PMA stimulation caused only a minor increase in their expression (Figure 7B). Importantly, in overexpressing tescalcin K562-Tsc(+) cells the basal levels of Ets- 1 and Ets- 2 mRNA along with their target gene, GPIIb, was significantly higher. The level of GATA-1 mRNA in these cells was not affected (Figure 7C). PMA stimulation for only 18 hours did not have a significant effect on GPIIb or Ets- 2 mRNA levels in control cells, although their expression was induced almost 4-fold in cells overexpressing tescalcin (Figure 7C). Downregulation of GATA-1 upon PMA stimulation is typical for K562 (52).

Taken together, our results demonstrate that expression levels of tescalcin in K562 and HEL cells regulate their megakaryocytic differentiation by controlling the genes encoding transcription factors of the Ets family.

\section{Discussion}

In the process of hematopoiesis, commitment and differentiation of progenitor cells along a particular lineage requires coordinated activation of cytokine receptor signaling pathways and cell-specific gene expression. In this study we report the discovery of a new factor necessary for megakaryocytic differentiation and expression of transcription factors of the Ets family.

Tescalcin is upregulated in megakaryocytic differentiation. Tescalcin was originally discovered as a novel gene temporally expressed in the area of the urogenital ridge and mesonephros (32). Although tescalcin has been implicated in the formation of male gonads, its function in gonadal development remains unknown. Furthermore, the urogenital ridge and mesonephros are the segments of aorta gonad mesonephros region, a potent site of hematopoiesis within the mammalian embryo and the first place from which hemato- 

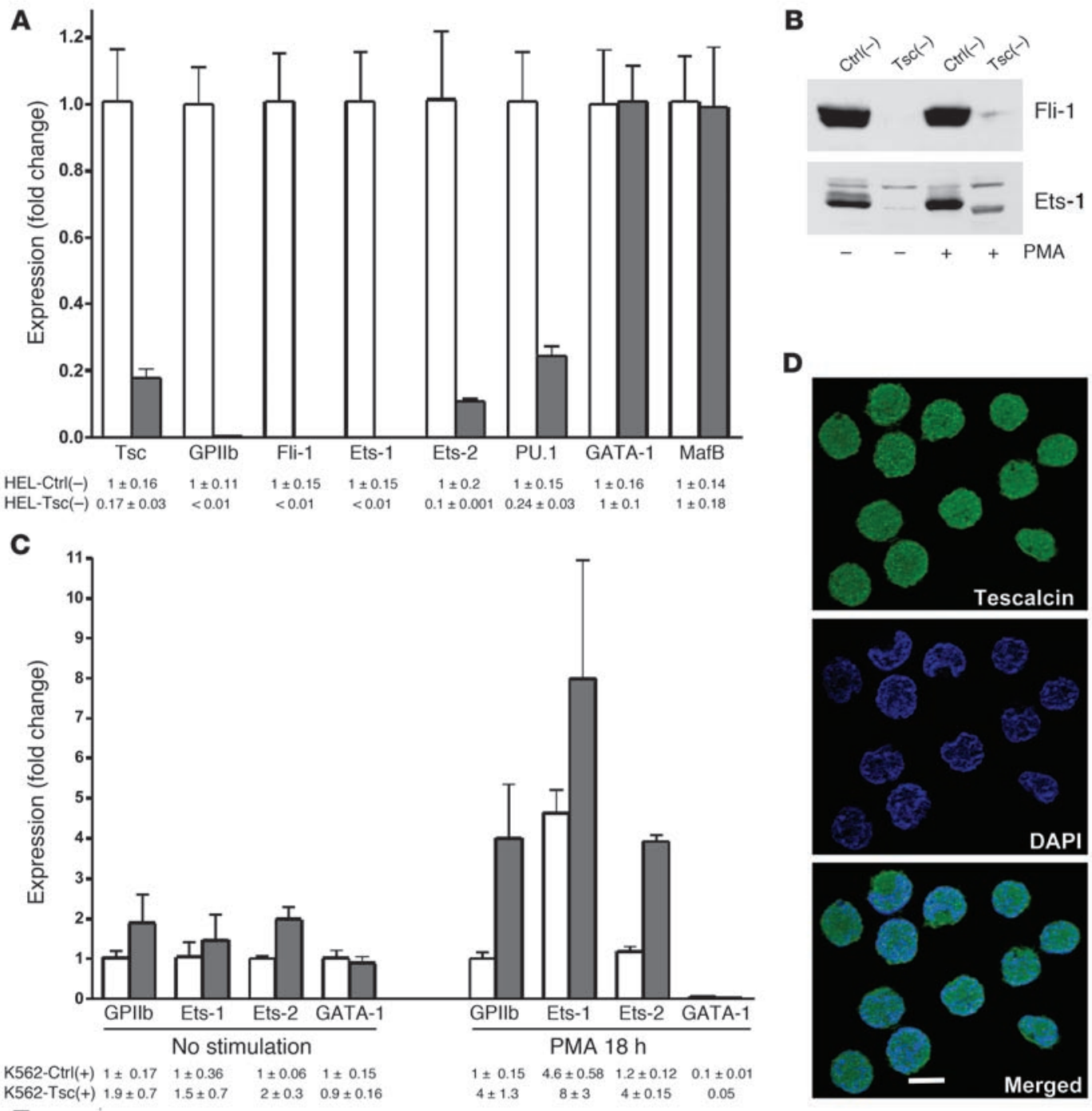

E

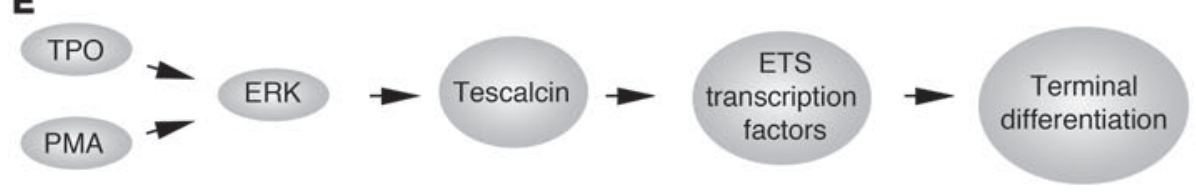

\section{Figure 7}

Tescalcin controls expression of Ets family transcription factors at the mRNA level. (A) Total RNA was isolated from HEL-Ctrl(-) (white bars) and HEL-Tsc(-) cells (gray bars). Gene expression was analyzed by RQ-PCR (TaqMan) with tescalcin-, GPIlb-, Ets-1-, Ets-2-, Fli-1-, PU.1-, GATA-1-, and MafB-specific primers as described in Methods. Data are expressed relative to wild-type HEL cells $(n=3$; mean \pm SD). (B) Control HEL-Ctrl(-) and tescalcin knockdown HEL-Tsc(-) cells were cultured in absence or presence of PMA for 72 hours. Expression of Ets-1 and Fli-1 transcription factors was assessed by Western blot with specific antibodies. (C) Total RNA was isolated from K562-Ctrl(+) and tescalcin overexpressing K562-Tsc(+) cells cultured in absence or presence of PMA for 18 hours. RQ-PCR using GPIlb-, Ets-1-, Ets-2-, and GATA-1-specific primers was performed as described in A. Data are expressed relative to wild-type unstimulated K562 $(n=3$; mean \pm SD). (D) Cytospin preparations of K562 cells on glass slides were stained with antibody to tescalcin and then with FITC-labeled donkey anti-rabbit antibody. Slides were mounted with DAPI containing mounting media (Prolong; Molecular Probes) to identify cell nuclei. All images were acquired at $\times 1,000$ magnification. Scale bar: $10 \mu \mathrm{m}$. (E) Outline of tescalcin-mediated pathway. TPO binds to its receptor, c-Mpl, on the cell surface and activates the ERK signaling cascade. Treatment of cells with PMA leads to a similar activation of ERK. The sustained ERK activity causes upregulation of tescalcin, which in turn promotes expression of the Ets family genes, orchestrating terminal differentiation along megakaryocytic lineage.

poietic stem cells emerge (53). A more detailed examination of tescalcin tissue distribution revealed that its mRNA is also expressed in other areas of hematopoiesis, such as fetal liver and adult bone marrow (33). In this study we show that tescalcin is expressed in the pluripotent hematopoietic progenitor cell lines K562 and HEL and its expression is upregulated during megakaryocytic differentiation. These findings led us to hypothesize that tescalcin plays a role in embryonic and adult hematopoiesis. 
K562 and HEL cell lines are widely used as models to study hematopoietic differentiation. They have an advantage over primary cells of being homogeneous and easily cultured and transfected, facilitating studies and analysis. Treatment of these cells with PMA induces typical features of megakaryocytic differentiation, such as growth arrest, progressive polyploidization, and acquisition of lineage-specific markers. Polyploidization (endomitosis), a unique feature of megakaryocytic cells, is characterized by repeated rounds of DNA replication without completion of anaphase B and cytokinesis (54). The beginning of endomitosis coincides with suppression of proliferation-promoting genes and temporary arrest at G0 phase. The ploidy of MKs increases progressively from $8 \mathrm{~N}$ up to $128 \mathrm{~N}$ and directly correlates with the ability to produce platelets (55). Examination of the cyclin machinery has shown that elevated levels of cyclin D3 and the associated kinase activity promote multiple cycles of endomitotic DNA synthesis (54). We have observed that expression of tescalcin is strongly increased during PMA-induced megakaryocytic differentiation of K562 and HEL cells (Figure 1, A and $\mathrm{B}$ ) as well as during TPO-induced maturation of primary MKs (Figure 1C). It is known that the differentiation and proliferative capacities of K562 cells correlate with the activation and localization of PKC- $\alpha$ and $-\beta$ II isoforms (56). PMA-induced megakaryocytic differentiation and cytostasis are accompanied by an increase in PKC- $\alpha$, a decrease in PKC- $\beta$ II levels, and sustained activation of the Raf/MEK/ERK signaling pathway. In contrast, bryostatin causes upregulation of both $\alpha$ and $\beta$ II isoforms and nuclear translocation of $\beta$ II. In K562 cells, this nuclear activity of PKC- $\beta$ II generates a proliferative signal that overrides the PMA-induced differentiation (40). In our experiments, bryostatin effectively inhibited PMAinduced upregulation of tescalcin, which supported our model that tescalcin upregulation correlates with megakaryocytic differentiation, and was not required for cell proliferation (Figure 1E). We also determined that this upregulation is mediated by sustained ERK activity. Only prolonged (>48 hours) stimulation of cells with PMA leads to an increase in tescalcin accumulation (Figure 1D). Pretreatment of cells with the specific MEK1/2 inhibitor U0126 abolished PMA-induced activation of the ERK1/2 kinase pathway as well as upregulation of tescalcin (Figure $1 \mathrm{~F}$ ). Together these results demonstrate that upregulation of tescalcin occurs downstream of ERK1/2 activation and led us to hypothesize that tescalcin is one of the causal factors in differentiation.

Tescalcin is essential for megakaryocytic differentiation. We manipulated the expression levels of tescalcin in K562 and HEL cells as well as in human bone marrow $\mathrm{CD} 34^{+}$progenitors. The overexpression of tescalcin in K562 cells was sufficient to induce all the tested traits of megakaryocytic differentiation. Surface expression of GPIIb in unstimulated cells was increased. The number of cells with $8 \mathrm{~N}$ and higher ploidy increased up to 10-fold, and the progression of polyploidization in response to PMA was significantly accelerated. Accumulation of cyclin D3, which is known to promote endomitosis in mouse MKs (43), was also increased. As expected of a differentiating cell, the rate of proliferation was notably decreased in K562, whereas HEL cells transfected with tescalcin expression construct could not grow at all. Downregulation of tescalcin in K562 and HEL cells produced an exactly opposite phenotype: increased proliferation and suppressed endomitosis under both resting and PMA-induced conditions. Cell adhesion and expression of GPIIb were blocked. The manifestation of these phenotypes was much stronger in HEL cells, perhaps because they have a higher basal level of endogenous tescalcin than do K562 cells.
In primary human bone marrow $\mathrm{CD} 34^{+}$cells, knockdown of tescalcin also resulted in phenotype consistent with its role in $\mathrm{MK}$ differentiation. Bone marrow $\mathrm{CD} 34^{+}$progenitors contain multiple types of CFUs that give rise to different hematopoietic lineages. Within the MK lineage there is a clear hierarchy of progenitor cells that determines their developmental and physical properties (57-60). The most primitive progenitors, BFU-MKs, have a higher proliferative capacity than the later, more committed CFU-MK. We observed that tescalcin knockdown led to a decrease in the number of large BFU-MK-derived colonies and a dramatic increase in a number of small CFU-MK-derived colonies. These CFU-MK colonies had unusually small number of cells (Figure 6, B and C). We have also observed the appearance of a large number of colonies that were negative for GPIIb staining. These distinct effects of the tescalcin knockdown can be explained by the inherent heterogeneity of $\mathrm{CD} 34^{+}$progenitors. It is possible that the phenotypic differences between progenitor subpopulations arise from dynamic changes in their gene expression status and different responses to stimuli. Tescalcin knockdown, therefore, can invoke qualitatively and/or quantitatively different effects on $\mathrm{CD} 34^{+}$subpopulations. To gain insight into how tescalcin mediates megakaryopoiesis at the molecular level, we used K562 and HEL cells, which are homogeneous, and focused on the regulation of GPIIb expression, a marker of megakaryocytic differentiation.

Tescalcin controls the expression of Ets family transcription factors. We demonstrate that in tescalcin-deficient cells both the accumulation of GPIIb mRNA and the activity of the GPIIb promoter were severely impaired (Figure 7A and Supplemental Figure 3). These facts imply that tescalcin controls the mechanism of GPIIb transcription.

It was known that expression of GPIIb, a classical megakaryocytic marker, is regulated through various response elements in its gene promoter and by combinatorial action of multiple transcriptional factors. Essential for megakaryocytopoiesis, transcription factor GATA-1 regulates transcription of GPIIb (51). The zinc-finger protein FOG and transcription factor AML-1/RUNX1 enhance the activity of GPIIb promoter through cooperation and physical interaction with GATA-1 $(61,62)$. Several transcription factors of the Ets family such as Fli-1, Ets-1, Ets-2, and PU.1 were also shown to transactivate GPIIb promoter (47-50). A member of the Maf family of transcription factors, MafB/Kreisler, regulates GPIIb promoter in a synergistic interaction between GATA-1 and Ets-1 (41). In our study the knockdown of tescalcin in HEL and K562 cells exerted a strong inhibitory effect on transcription of genes encoding members of the Ets family, particularly Fli-1, Ets-1, and Ets-2. In agreement with this, overexpression of tescalcin in K562-Tsc(+) cells raised basal levels of Ets- 1 and Ets- 2 mRNAs along with their target, GPIIb. The levels of GATA-1 mRNA remained unchanged. Moreover, high levels of tescalcin in K562-Tsc(+) cells allowed for a faster transcriptional upregulation of marker genes after a short treatment with inducer.

These findings allow us to outline a pathway in which upregulation of tescalcin by the ERK signaling cascade promotes the expression of Ets family transcription factors and induces the events of megakaryocytic differentiation (Figure 7E). The exact molecular mechanisms of how tescalcin regulates the expression of transcription factors and how transcription of tescalcin itself is regulated remain to be elucidated. Tescalcin does not possess a DNA binding domain, however it potentially can regulate gene expression via one of the mechanisms known for other EF-hand $\mathrm{Ca}^{2+}$-binding proteins. For example, calmodulin-dependent protein kinases acti- 
vated by $\mathrm{Ca}^{2+} /$ calmodulin phosphorylate various protein substrates including MAPKs and CREB. This results in the activation of transcriptional coactivator $\mathrm{CBP} / \mathrm{p} 300$, which is known to regulate chromatin remodeling, and numerous transcription factors including those involved in hematopoiesis (63-65). Calmodulin also regulates protein phosphatase calcineurin, which controls translocation of transcription factor NFAT from cytosol to the nucleus (66). Alternatively, some EF-hand proteins can directly interact with transcription factors within the nucleus. Some members of the S100 subfamily of EF-hand proteins can either stimulate or inhibit the activity of the tumor suppressor p53 by direct interaction and alteration of its oligomerization and phosphorylation states $(67,68)$. Direct binding of DNA represents yet another mechanism of gene expression regulation recently discovered for EF-hand proteins. Neuronal transcriptional repressor DREAM, which does not have a distinct DNA binding domain, nevertheless binds DNA regulatory elements downstream of some promoter sequences. At low $\mathrm{Ca}^{2+}$ concentration DREAM forms a tetramer capable of specific DNA binding, whereas high $\mathrm{Ca}^{2+}$ concentration induces formation of a dimer, which is incapable of DNA binding and transcriptional repression $(69,70)$. Previous studies $(33,35)$ reported the interaction of tescalcin with calcineurin and $\mathrm{Na}^{+} / \mathrm{H}^{+}$exchanger, but it is not yet clear whether these in vitro studies are physiologically significant. In fact, a pool of endogenous tescalcin is found in the nucleus of K562 cells (Figure 7D), where it may exert functions related to transcription, chromatin remodeling, and other nuclear activities. Since tescalcin undergoes a distinct conformation change at submicromolar $\mathrm{Ca}^{2+}$ (33), it is tempting to speculate that it might work as a "coincidence detector" responding to transient changes in free $\mathrm{Ca}^{2+}$, only when the sustained activation of the MAPK pathway by growth factors (Figure 7E) increases tescalcin expression.

Transcription factors of the Ets family regulate the expression of genes involved in cellular proliferation, differentiation, development, transformation, and apoptosis $(9,11)$, which may explain the magnitude of the effect of tescalcin knockdown on megakaryocytic differentiation. Besides hematopoiesis, Ets signal transduction is also essential in angiogenesis/vasculogenesis at the earliest embryonic stages and is later involved in tissue development. In adult animals, Ets genes are expressed in multiple tissues, including central nervous system, ovaries, testes, kidneys, and lungs (71). Since tescalcin is also highly expressed in several tissues including heart and brain, it is reasonable to expect that it plays an important role in terminal differentiation of many cell types.

\section{Methods}

Cell culture, transfection, stable cell lines, and luciferase assay. K562 (CCL-243) and HEL (TIB-180) cells were supplied by the ATCC and cultured in RPMI with $10 \% \mathrm{FCS}$ and $1 \times$ penicillin/streptomycin at $37^{\circ} \mathrm{C}, 95 \%$ humidity, and $5 \% \mathrm{CO}_{2}$ pressure. For transfection, the exponentially growing $\mathrm{K} 562$ or HEL cells were washed twice with PBS and resuspended in serum-free RPMI media at concentration $2 \times 10^{7}$ cells $/ \mathrm{ml}$. The electroporation was performed using $80 \mu \mathrm{g}$ of plasmid DNA mixed with $0.8 \mathrm{ml}$ of cell suspension. Cells were subjected to a single electric pulse (960 microfarad, $250 \mathrm{~V})$ in $0.4-\mathrm{cm}$ gap electroporation cuvettes using a Bio-Rad Gene Pulser electroporator, followed by dilution to $5 \times 10^{5}$ cells $/ \mathrm{ml}$ in RPMI 1640 (Invitrogen) with $20 \%$ FCS and antibiotics. For making stable cell lines, neomycin-resistant cells were selected by G418 (1.5 mg/ml; Invitrogen) for 2 weeks and then cloned by limited dilution in 96-well plates. Single-cell clones were expanded and screened for the expression of tescalcin by western blot analysis. Selected clones were maintained in RPMI with $10 \% \mathrm{FCS}$ and $1 \mathrm{mg} / \mathrm{ml}$ of G418.
Purification of primary MKs. The procedure for isolation and culture of mouse MKs was essentially described in ref. 39 . Briefly, whole livers were recovered from mouse fetuses on 13-15 dpc, and single-cell suspensions were prepared by repeated passage through 21 - and 25 -gauge needles. Fetal liver cells were then cultured in DMEM with $10 \%$ FCS, 2 mM L-glutamine, $0.5 \times$ penicillin/streptomycin, $0.1 \mathrm{mM}$ nonessential amino acids, and 0.1 $\mu \mathrm{g} / \mathrm{ml}$ TPO. Mature MKs were harvested on the fifth and eighth days of culture and purified on a 1-step BSA gradient as described in ref. 72 . The cell suspension was loaded onto $1.5 \%$ over $3 \%$ BSA and sedimented at normal gravity for 45 minutes at room temperature. The bottom cell fraction contained more than $90 \%$ large cholinesterase-positive cells. This was determined essentially as described in ref. 73 .

Design and characterization of tescalcin-specific shRNA. The sequence targeting nucleotides 188-208 of tescalcin open reading frame was determined by GenScript siRNA Target Finder software (http://www.genscript.com/ ssl-bin/app/rnai) and subjected to a BLAST search (http://www.ncbi.nlm. nih.gov/BLAST/) against the NCBI human Non-RefSeq RNA library to ensure that no other gene(s) were targeted. The design of the negative control construct pshSCR was based on the rearranged (scrambled) tescalcin target sequence. Pairs of complementary synthetic oligonucleotides for the tescalcin target sequences 5'-GATCCCGTGTTGTCGAAGAAGGCACGAATTGATATCCGTTCGTGCCTTCTTCGACAACATTTTTTCCAAA-3' (shTSC-A), 5'-AGCTTTTGGAAAAAATGTTGTCGAAGAAGGCACGAACGGATATCAATTCGTGCCTTCTTCGACAACACGG-3' (shTSC-B), 5'-GATCCCGGTGATTCCTGAACTGCCGTTGTTGATATCCGCAACGGCAGTTCAGGAATCACTTTTTTCCAAA-3' (shSCR-A), and 5'-AGCTTTTGGAAAAAAGTGATTCCTGAACTGCCGTTGCGGATATCAACAACGGCAGTTCAGGAATCACCGG-3' (shSCR-B) were annealed together and cloned into pRNA-U6.1/Neo vector (GenScript Corp.) to generate plasmids psiTSC and psiSCR, respectively. The specificity and efficiency of psiTSC was evaluated in the pilot experiment when HeLa cells were transiently cotransfected with pTSC, pshTSC, and pshSCR plasmid DNAs, in which pshTSC was able to markedly suppress the overexpression of tescalcin driven by pTSC.

RNA isolation and quantification of gene expression. Total RNA was extracted from cells using TRIzOL reagent (Invitrogen), purified over RNeasy column with DNase digestion step (QIAGEN), and converted to cDNA using High-Capacity Reverse Transcription kit (Applied Biosystems). RQ-PCR was performed using 10 or 20 ng of cDNA, TaqMan Fast Universal PCR Master Mix, and the following Taqman Gene Expression Assays: Ets-1 (Hs00428287_m1), Ets-2 (Hs00232009_m1), Fli-1 (Hs00231107_m1), GATA-1 (Hs00231112_m1), GPIIb (Hs00166246_m1), MafB (Hs00534343_s1), PU.1 (Hs02786711_m1), tescalcin (Hs00215487_m1), and 18S (4333760T). The reactions were run in triplicate on the 7900HT Fast Real-Time PCR System (Applied Biosystems) and normalized to the endogenous control $18 \mathrm{~S}$ ribosomal RNA.

Lentiviral vector construction and production. The lentiviral transfer vectors were generated as follows. A portion of pshTSC or pshSCR plasmid containing U6 promoter and shRNA target sequence was inserted into pRRLsin. cPPT.hCMV.EGFP.Wpre vector (74) upstream of the CMV promoter. For production of lentivirus, $293 \mathrm{~T}$ cells were cotransfected with transfer vectors pCMVdR8.74 and pMD2VSV.G essentially as described in refs. 75, 76. Lentiviruses were harvested 72 hours after transfection, passed through a $0.45-\mu \mathrm{M}$ filter, and concentrated 100 -fold by ultracentrifugation through $20 \%$ sucrose cushion $\left(100,000 \mathrm{~g}\right.$ for 90 minutes; $\left.4^{\circ} \mathrm{C}\right)$. Titers of viral stock were determined on HEL cells transduced by spinoculation (1,000 $\mathrm{g}$ for 90 minutes; $30^{\circ} \mathrm{C}$ ) and analyzed by FACS 48 hours later. The efficacy of tescalcin knockdown in HEL cells was determined by western blotting.

In vitro transduction of bone marrow cells and CFU-MK assay. Human bone marrow $\mathrm{CD}_{3}{ }^{+}$progenitor cells were purchased from Stem Cell Technologies Inc. Cells were suspended at $10^{5}$ cells $/ \mathrm{ml}$ in serum-free media 
(Stemline II; Sigma-Aldrich) with $8 \mu \mathrm{g} / \mathrm{ml}$ polybrene and transduced by spinoculation (MOI $=5$ ), followed by wash and 16 hours recovery in Stemline II media. To determine transduction efficiency, cells were cultured in Stemline II media, supplemented with cytokines (100 ng/ml TPO; $100 \mathrm{ng} /$ $\mathrm{ml} \mathrm{SCF} ; 100 \mathrm{ng} / \mathrm{ml}$ G-CSF) for an additional 72 hours, and then analyzed by FACS for GFP expression. For megakaryocytic differentiation, $5 \times 10^{3}$ CD $34^{+}$cells were cultured on chamber slides by means of a commercially available kit (MegaCult-C; StemCell Technologies). After 12 days in culture, cells on slides were fixed, immunolabeled for GPIIb/IIIa, and scored under microscope. Three categories of colonies were identified: pure MK colonies, mixed MK colonies (distinguished by the presence of non-MK cells), and non-MK colonies. Pure MK colonies were scored according to size and maturity as follows: large BFU-MKs with more than 50 cells and 2 or more different foci of development; medium BFU-MK colonies with 20-50 cells; and small CFU-MKs with 3-20 cells.

Statistics. Statistical analysis was performed using paired, 2-tailed Student's $t$ test and Microsoft Office Excel 2003 software. Statistical significance was accepted at the $P<0.05$ level.

1. Alberola-Ila, J., Forbush, K.A., Seger, R., Krebs, E.G., and Perlmutter, R.M. 1995. Selective requirement for MAP kinase activation in thymocyte differentiation. Nature. 373:620-623.

2. Sharp, L.L., Schwarz, D.A., Bott, C.M., Marshall, C.J., and Hedrick, S.M. 1997. The influence of the MAPK pathway on $\mathrm{T}$ cell lineage commitment. Immunity. 7:609-618.

3. Miranda, M.B., McGuire, T.F., and Johnson, D.E. 2002. Importance of MEK-1/-2 signaling in monocytic and granulocytic differentiation of myeloid cell lines. Leukemia. 16:683-692.

4. Herrera, R., Hubbell, S., Decker, S., and Petruzzelli, L. 1998. A role for the MEK/MAPK pathway in PMA-induced cell cycle arrest: modulation of megakaryocytic differentiation of K562 cells. Exp. Cell Res. 238:407-414.

5. Melemed, A.S., Ryder, J.W., and Vik, T.A. 1997. Activation of the mitogen-activated protein kinase pathway is involved in and sufficient for megakaryocytic differentiation of CMK cells. Blood. 90:3462-3470.

6. Racke, F.K., Lewandowska, K., Goueli, S., and Goldfarb, A.N. 1997. Sustained activation of the extracellular signal-regulated kinase/mitogenactivated protein kinase pathway is required for megakaryocytic differentiation of K562 cells. J. Biol. Chem. 272:23366-23370.

7. Uchida, M., et al. 2001. A functional role of mitogen-activated protein kinases, erk1 and erk2, in the differentiation of a human leukemia cell line, UT-7/ GM: a possible key factor for cell fate determination toward erythroid and megakaryocytic lineages. Int. J. Hematol. 73:78-83.

8. Whalen, A.M., Galasinski, S.C., Shapiro, P.S., Nahreini, T.S., and Ahn, N.G. 1997. Megakaryocytic differentiation induced by constitutive activation of mitogen-activated protein kinase kinase. Mol. Cell. Biol. 17:1947-1958.

9. Sharrocks, A.D. 2001. The ETS-domain transcription factor family. Nat. Rev. Mol. Cell Biol. 2:827-837.

10. Wasylyk, B., Hagman, J., and Gutierrez-Hartmann, A. 1998. Ets transcription factors: nuclear effectors of the Ras-MAP-kinase signaling pathway. Trends Biochem. Sci. 23:213-216.

11. Sementchenko, V.I., and Watson, D.K. 2000. Ets target genes: past, present and future. Oncogene. 19:6533-6548

12. Yordy, J.S., and Muise-Helmericks, R.C. 2000. Signal transduction and the Ets family of transcription factors. Oncogene. 19:6503-6513.

13. Bastian, L.S., Kwiatkowski, B.A., Breininger, J., Danner, S., and Roth, G. 1999. Regulation of the megakaryocytic glycoprotein IX promoter by the

\section{Acknowledgments}

We thank Beatriz M.A. Fontoura, Joy Lincoln, Robert B. Levy, K. Saidas Nair, and Vijaya Narayanan for many helpful suggestions on the manuscript. We thank Agata Levay for help with mouse embryos and James Phillips for assistance with data acquisition. This work was supported by the University of Miami Sylvester Cancer Center development grant (to K. Levay) and NIH grants GM 060019 and NEI 012982 (to V.Z. Slepak).

Received for publication November 21, 2005, and accepted in revised form June 5, 2007.

Address correspondence to: Konstantin Levay or Vladlen Z. Slepak, Department of Molecular and Cellular Pharmacology, University of Miami Miller School of Medicine, PO Box 016189 (R-189), Miami, Florida 33101-6189, USA. Phone: (305) 243-3431; Fax: (305) $243-$ 4555; E-mail: k.levay@miami.edu (K. Levay). Phone: (305) 243-3430; Fax: (305) 243-4555; E-mail: v.slepak@miami.edu (V.Z. Slepak). oncogenic Ets transcription factor Fli-1. Blood. 93:2637-2644.

14. Bastian, L.S., Yagi, M., Chan, C., and Roth, G.J. 1996. Analysis of the megakaryocyte glycoprotein IX promoter identifies positive and negative regulatory domains and functional GATA and Ets sites. J. Biol. Chem. 271:18554-18560.

15. Deveaux, S., et al. 1996. Analysis of the thrombopoietin receptor (MPL) promoter implicates GATA and Ets proteins in the coregulation of megakaryocyte-specific genes. Blood. 87:4678-4685.

16. Eisbacher, M., et al. 2003. Protein-protein interaction between Fli-1 and GATA-1 mediates synergistic expression of megakaryocyte-specific genes through cooperative DNA binding. Mol. Cell. Biol. 23:3427-3441.

17. Eisbacher, M., Khachigian, L.M., Khin, T.H., Holmes, M.L., and Chong, B.H. 2001. Inducible expression of the megakaryocyte-specific gene glycoprotein IX is mediated through an Ets binding site and involves upstream activation of extracellular signalregulated kinase. Cell Growth Differ. 12:435-445.

18. Hashimoto, Y., and Ware, J. 1995. Identification of essential GATA and Ets binding motifs within the promoter of the platelet glycoprotein Ib alpha gene. J. Biol. Chem. 270:24532-24539.

19. Holmes, M.L., Bartle, N., Eisbacher, M., and Chong, B.H. 2002. Cloning and analysis of the thrombopoietin-induced megakaryocyte-specific glycoprotein VI promoter and its regulation by GATA-1, Fli-1, and Sp1. J. Biol. Chem. 277:48333-48341.

20. Lepage, A., et al. 1999. Functional characterization of the human platelet glycoprotein $\mathrm{V}$ gene promoter: a specific marker of late megakaryocytic differentiation. Blood. 94:3366-3380.

21. Mignotte, V., et al. 1994. Structure and transcription of the human c-mpl gene (MPL). Genomics. 20:5-12.

22. Minami, T., Tachibana, K., Imanishi, T., and Doi, T. 1998. Both Ets-1 and GATA-1 are essential for positive regulation of platelet factor 4 gene expression. Eur. J. Biochem. 258:879-889.

23. Sato, N., et al. 2000. Functional conservation of platelet glycoprotein V promoter between mouse and human megakaryocytes. Exp. Hematol. 28:802-814.

24. Schwachtgen, J.L., et al. 1997. Ets transcription factors bind and transactivate the core promoter of the von Willebrand factor gene. Oncogene. 15:3091-3102.

25. Jackers, P., Szalai, G., Moussa, O., and Watson, D.K. 2004. Ets-dependent regulation of target gene expression during megakaryopoiesis. J. Biol. Chem. 279:52183-52190.
26. Ghozi, M.C., Bernstein, Y., Negreanu, V., Levanon, D., and Groner, Y. 1996. Expression of the human acute myeloid leukemia gene AML1 is regulated by two promoter regions. Proc. Natl. Acad. Sci. U. S. A. 93:1935-1940.

27. Gu, T.L., Goetz, T.L., Graves, B.J., and Speck, N.A. 2000. Auto-inhibition and partner proteins, corebinding factor beta (CBFbeta) and Ets-1, modulate DNA binding by CBFalpha2 (AML1). Mol. Cell. Biol. 20:91-103.

28. Ichikawa, M., et al. 2004. AML-1 is required for megakaryocytic maturation and lymphocytic differentiation, but not for maintenance of hematopoietic stem cells in adult hematopoiesis. Nat. Med. 10:299-304.

29. Hart, A., et al. 2000. Fli-1 is required for murine vascular and megakaryocytic development and is hemizygously deleted in patients with thrombocytopenia. Immunity. 13:167-177.

30. Spyropoulos, D.D., et al. 2000. Hemorrhage, impaired hematopoiesis, and lethality in mouse embryos carrying a targeted disruption of the Fli1 transcription factor. Mol. Cell. Biol. 20:5643-5652.

31. Raslova, H., et al. 2004. FLI1 monoallelic expression combined with its hemizygous loss underlies Paris-Trousseau/Jacobsen thrombopenia. J. Clin. Invest. 114:77-84. doi:10.1172/JCI200421197.

32. Perera, E.M., et al. 2001. Tescalcin, a novel gene encoding a putative $\mathrm{EF}-$ hand $\mathrm{Ca}(2+)$-binding protein, $\mathrm{Col9a} 3$, and renin are expressed in the mouse testis during the early stages of gonadal differentiation. Endocrinology. 142:455-463.

33. Gutierrez-Ford, C., et al. 2003. Characterization of tescalcin, a novel EF-hand protein with a single Ca2+-binding site: metal-binding properties, localization in tissues and cells, and effect on calcineurin. Biochemistry. 42:14553-14565.

34. Li, X., Liu, Y., Kay, C.M., Muller-Esterl, W., and Fliegel, L. 2003. The $\mathrm{Na}+\mathrm{H}+$ exchanger cytoplasmic tail: structure, function, and interactions with tescalcin. Biochemistry. 42:7448-7456.

35. Mailander, J., Muller-Esterl, W., and Dedio, J. 2001. Human homolog of mouse tescalcin associates with $\mathrm{Na}(+) / \mathrm{H}(+)$ exchanger type-1. FEBS Lett. 507:331-335

36. Leary, J.F., et al. 1987. Multipotent human hematopoietic cell line K562: lineage-specific constitutive and inducible antigens. Leuk. Res. 11:807-815.

37. Papayannopoulou, T., Yokochi, T., Nakamoto, B., and Martin, P. 1983. The surface antigen profile of HEL cells. Prog. Clin. Biol. Res. 134:277-292.

38. Long, M.W., Heffner, C.H., Williams, J.L., Peters, C., and Prochownik, E.V. 1990. Regulation of megakaryocyte phenotype in human erythroleukemia 
cells. J. Clin. Invest. 85:1072-1084.

39. Lecine, P., Blank, V., and Shivdasani, R. 1998. Characterization of the hematopoietic transcription factor NF-E2 in primary murine megakaryocytes. J. Biol. Chem. 273:7572-7578.

40. Hocevar, B.A., Morrow, D.M., Tykocinski, M.L., and Fields, A.P. 1992. Protein kinase $C$ isotypes in human erythroleukemia cell proliferation and differentiation. J. Cell Sci. 101:671-679.

41. Sevinsky, J.R., Whalen, A.M., and Ahn, N.G. 2004. Extracellular signal-regulated kinase induces the megakaryocyte GPIIb/CD41 gene through MafB/ Kreisler. Mol. Cell. Biol. 24:4534-4545.

42. Wang, Z., Zhang, Y., Kamen, D., Lees, E., and Ravid, K. 1995. Cyclin D3 is essential for megakaryocytopoiesis. Blood. 86:3783-3788.

43. Zimmet, J.M., Toselli, P., and Ravid, K. 1998. Cyclin D3 and megakaryocyte development: exploration of a transgenic phenotype. Stem Cells. 16(Suppl. 2):97-106.

44. Berthier, R., Jacquier-Sarlin, M., Schweitzer, A., Block, M.R., and Molla, A. 1998. Adhesion of mature polyploid megakaryocytes to fibronectin is mediated by beta 1 integrins and leads to cell damage. Exp. Cell Res. 242:315-327.

45. Coulombel, L., Auffray, I., Gaugler, M.H., and Rosemblatt, M. 1997. Expression and function of integrins on hematopoietic progenitor cells. Acta Haematol. 97:13-21.

46. Emambokus, N.R., and Frampton, J. 2003. The glycoprotein IIb molecule is expressed on early murine hematopoietic progenitors and regulates their numbers in sites of hematopoiesis. Immunity. 19:33-45.

47. Athanasiou, M., et al. 1996. Increased expression of the ETS-related transcription factor FLI-1/ERGB correlates with and can induce the megakaryocytic phenotype. Cell Growth Differ. 7:1525-1534.

48. Block, K.L., Shou, Y., and Poncz, M. 1996. An Ets/ Sp1 interaction in the $5^{\prime}$-flanking region of the megakaryocyte-specific alpha IIb gene appears to stabilize Sp1 binding and is essential for expression of this TATA-less gene. Blood. 88:2071-2080.

49. Doubeikovski, A., et al. 1997. Thrombopoietininduced expression of the glycoprotein IIb gene involves the transcription factor PU.1/Spi-1 in UT7-Mpl cells. J. Biol. Chem. 272:24300-24307.

50. Lemarchandel, V., Ghysdael, J., Mignotte, V., Rahuel, C., and Romeo, P.H. 1993. GATA and Ets cis-acting sequences mediate megakaryocyte-specific expression. Mol. Cell. Biol. 13:668-676.

51. Martin, F., Prandini, M.H., Thevenon, D., Marguerie, G., and Uzan, G. 1993. The transcription factor GATA-1 regulates the promoter activity of the platelet glycoprotein IIb gene. J. Biol. Chem. 268:21606-21612.

52. Dai, W., and Murphy, M.J., Jr. 1993. Downregulation of GATA-1 expression during phorbol myristate acetate-induced megakaryocytic differentiation of human erythroleukemia cells. Blood. 81:1214-1221.

53. Dzierzak, E. 2005. The emergence of definitive hematopoietic stem cells in the mammal. Curr. Opin. Hematol. 12:197-202.

54. Ravid, K., Lu, J., Zimmet, J.M., and Jones, M.R. 2002. Roads to polyploidy: the megakaryocyte example. J. Cell. Physiol. 190:7-20.

55. Mattia, G., et al. 2002. Different ploidy levels of megakaryocytes generated from peripheral or cord blood CD34+ cells are correlated with different levels of platelet release. Blood. 99:888-897.

56. Murray, N.R., Baumgardner, G.P., Burns, D.J., and Fields, A.P. 1993. Protein kinase C isotypes in human erythroleukemia (K562) cell proliferation and differentiation. Evidence that beta II protein kinase $\mathrm{C}$ is required for proliferation. J. Biol. Chem. 268:15847-15853.

57. Briddell, R.A., Brandt, J.E., Straneva, J.E., Srour, E.F., and Hoffman, R. 1989. Characterization of the human burst-forming unit-megakaryocyte. Blood. 74:145-151.

58. Hoffman, R. 1989. Regulation of megakaryocytopoiesis. Blood. 74:1196-1212.

59. Levin, J., Levin, F.C., Penington, D.G., and Metcalf, D. 1981. Measurement of ploidy distribution in megakaryocyte colonies obtained from culture: with studies of the effects of thrombocytopenia. Blood. 57:287-297.

60. Long, M.W., Gragowski, L.L., Heffner, C.H., and Boxer, L.A. 1985. Phorbol diesters stimulate the development of an early murine progenitor cell. The burst-forming unit-megakaryocyte. J. Clin. Invest. 76:431-438.

61. Elagib, K.E., et al. 2003. RUNX1 and GATA-1 coexpression and cooperation in megakaryocytic differentiation. Blood. 101:4333-4341.

62. Gaines, P., Geiger, J.N., Knudsen, G., Seshasayee, D., and Wojchowski, D.M. 2000. GATA-1- and FOG- dependent activation of megakaryocytic alpha IIB gene expression. J. Biol. Chem. 275:34114-34121.

63. Blobel, G.A. 2000. CREB-binding protein and p300: molecular integrators of hematopoietic transcription. Blood. 95:745-755.

64. Blobel, G.A. 2002. CBP and p300: versatile coregulators with important roles in hematopoietic gene expression. J. Leukoc. Biol. 71:545-556.

65. Mellstrom, B., and Naranjo, J.R. 2001. Mechanisms of $\mathrm{Ca}(2+)$-dependent transcription. Curr. Opin. Newrobiol. 11:312-319.

66. Ikura, M., Osawa, M., and Ames, J.B. 2002. The role of calcium-binding proteins in the control of transcription: structure to function. Bioessays. 24:625-636.

67. Fernandez-Fernandez, M.R., Veprintsev, D.B., and Fersht, A.R. 2005. Proteins of the S100 family regulate the oligomerization of p53 tumor suppressor. Proc. Natl. Acad. Sci. U. S. A. 102:4735-4740.

68. Mueller, A., et al. 2005. The calcium-binding protein S100A2 interacts with p53 and modulates its transcriptional activity. J. Biol. Chem. 280:29186-29193.

69. Carrion, A.M., Link, W.A., Ledo, F., Mellstrom, B., and Naranjo, J.R. 1999. DREAM is a Ca2+-regulated transcriptional repressor. Nature. 398:80-84.

70. Osawa, M., et al. 2005. Mg2+ and Ca2+ differentially regulate DNA binding and dimerization of DREAM. J. Biol. Chem. 280:18008-18014.

71. Maroulakou, I.G., and Bowe, D.B. 2000. Expression and function of Ets transcription factors in mammalian development: a regulatory network. Oncogene. 19:6432-6442.

72. Drachman, J.G., Sabath, D.F., Fox, N.E., and Kaushansky, K. 1997. Thrombopoietin signal transduction in purified murine megakaryocytes. Blood. 89:483-492.

73. Jackson, C.W. 1973. Cholinesterase as a possible marker for early cells of the megakaryocytic series. Blood. 42:413-421.

74. Follenzi, A., Ailles, L.E., Bakovic, S., Geuna, M., and Naldini, L. 2000. Gene transfer by lentiviral vectors is limited by nuclear translocation and rescued by HIV-1 pol sequences. Nat. Genet. 25:217-222.

75. Dull, T., et al. 1998. A third-generation lentivirus vector with a conditional packaging system. J. Virol. 72:8463-8471.

76. Naldini, L., et al. 1996. In vivo gene delivery and stable transduction of nondividing cells by a lentiviral vector. Science. 272:263-267. 